\title{
On the Existence of Undiscovered Uranian Satellites
}

Daniel C. Boice 
ON THE EXISTENCE OF UNDISCOVERED URANIAN SATELLITES

by

Danie1 C. Boice

\begin{abstract}
Structure in the Uranian ring system as observed in recent occultations may contain indirect evidence for the existence of undiscovered satellites. Using the Alfvén and Arrhenius (1975, 1976) scenario for the formation of planetary systems, the orbital radii of up to nine hypothetical satellites interior to Miranda are computed. These calculations should provide interesting comparisons when the results from the Voyager 2 encounter with Uranus are made public.
\end{abstract}

\title{
I. INTRODUCTION
}

Many interesting ideas have been suggested to explain the structure and stability of the ririg around Saturn and Uranus. In each of these theories, many details of the ring structures have been interpreted as resulting from: two-body resonances (Goldreich and Tremaine, 1978; Holbert, et a1., 1982; Lissauer and Cuzzi, 1982), shepherd satellites (Goldreich and Tremaine, 1979; Synnott et al., 1983; Borderies, 1984) three-body resonances (Patterson, 1986), or modern Laplacian theory (Prentice, 1986). However, each of these ideas has its relative merits and difficulties. Nearly five years after the Voyager 2 encounter with Saturn, a comprehensive explaration of the ring structure does not exist and many mysteries remain. 
More than a decade ago, Alfvén and Arrhenius (1975, 1976) proposed an alternate explanation of the formation of the solar system that incorporated plasma processes thought to be dominant in an early epoch of cosmogony. They have applied their model (AA hereafter) to Saturn's rings to interpret many structures such as Cassini's division and double ringlets. The AA model postulates a causal relationship between the location of Saturn's nearest moons to the ring minima that resulted from the formation process. Despite some successes, their modei remains controversial and has not gained wide acceptance in the literature.

With the discovery of the Uranian ring system (Elliot et al., 1977), it is possible to invoke the AA scenario by assuming that the minima in the Uranian ring system were caused by the effects of nearby satellites and to solve for their orbital radii. It is very unlikely that all ring minima can be accounted for in this manner but all were included for completeness. With the Voyager 2 encounter of Uranus in January 1986, these calculations are opportune.

In the remainder of this paper I give a summary of the AA model and discuss its implications for predicting new Uranian satelitites from observations of the Uranian ring structure.

\section{MODEL SUMMARY}

A complete discussion of the AA model can be found in Alfvén and Arrhenius (1975, 1976) and in Alfvén (1983) so only a brief summary, as it applies to planetary systems, is given below.

In the early formation of a planetary system like Saturn or Uranus, charged particles rotate around the planet in an axisymmetric dipole magnetic field. Their motion is dominated by the magnetic field such that a quasi- 
stationary situation exists. This requires that the gravity is balanced by the magnetic and centrifugal forces. At a certain evolutionary stage a transition from the plasma state to neutral planetesimals occurs, cancelling the magnetic forces. This results in a collapse to the equatorial plane and a contraction by a factor of approximately $2 / 3$ which is derived from geometric considerations of the dipole magnetic field. Initially the orbits of these planetesimals are elliptical but mutual collisions circularize them. Further absorption of plasma by the ring results in a small correction to the contraction factor which reduces it to about 0.64 .

AA postulate that the transition from plasma to planetesimal was a slow process. During this condensation epoch the formation of satellites by accreting planetesimals would deplete regions of material. The subsequent contraction of this material containing these voids would produce minima in the ring structure which AA label the "cosmogonic shadow effect." They cite three minima in the asteroid belt and numerous minima in the Saturnian rings, including Cassini's division (the "shadow" of Mimas), as successful interpretations of their model.

\section{APPLICATION TO THE URANIAN SYSTEM}

Discussing the application of this model to the Uranian system I assume that the observed ring minima are cosmogonic shadows of satellites. In this manner the satellite-ring minimum relationship has been inverted to speculate on the existence of new satellites from possible evidence contained in the structure of the rings. Surely, the presence of a ring minimum does not guarantee the existence of a moon but is only a possibility within the context of the AA model. 
It has been conjectured by Curtis and Ness (1985) that Uranus has no intrinsic magnetic field. If this does prove to be the case the following analysis is unaffected, as the AA model only requires the existence of a primordial magnetic field.

The predicted satellite orbital radii which are calculated by "scaling up" the minima by the AA contractior. factor, 0.64 , are presented in Table 1. These hypothetical moons have been tentatively labeled U1 through U9. Also shown are the ring radii adopted by Elilot et al. (1978) along with radius values for the midpoints of the ring minima. These hypothetical moons are located between the orbits of Miranda and the outermost $E$ ring. The five known satellites of Uranus are too distant to be responsible for ring minima as their "shadows" are outside of the Roche limit.

A diagram showing the location of the new satellites in the Uranian system is given in Figure 1. The length of each arrow pointing to the orbital radius of the hypothetical moon is a rough indication of the likelihood of its existence within the scope of the AA model. This estimation is based on the argument that the mass of a moon should be roughly proportional to the size of its shadow. In the case of the larger minima such as the $\delta-\varepsilon$ and the 4- $\alpha$ gap, a simple scaling in the above fashion from the Mimas-Cassini division relationship at Saturn gives a radius of approximately $230 \mathrm{~km}$ for a satellite responsible for the $\delta-\varepsilon$ minimum. A search for Uranian satellites conducted by Smith (1985) at Los Companos Observatory using a special coronagraph and a CCD camera was unsuccessful in detecting new satellites. Smith reports a radius of about $15 \mathrm{~km}$ as the observational limit of his search so that hypothetical moons responsible for the larger minima would have been detected. 
The relative error associated with the radii given in Table 1 and Figure 1 is 0.016 which corresponds to about $\pm 1150 \mathrm{~km}$ or $\pm 0.04 \mathrm{R}_{\mathrm{u}}$. This was estimated by using $\pm 100 \mathrm{~km}$ as the error in the location of the rings (E1liot et al., 1978) and \pm 0.01 as the uncertainty in the contraction factor (Alfvén, 1983).

If some of the minima in the Uranian rings are cosmogonic shadows then higher resolution images should show detailed structures such as double ringlets, explained in the AA model as "anti-shadow" features located on either side of the minima.

\section{SUMMARY}

Applying the AA model of the formation of planetary s stems to the structure of the Uranian rings, evidence for as many as nine new satellites has been postulated. The evidence is not yet compelling but does raise this intriguing possibility. This report is particuiarly timely as the Voyager 2 spacecraft encounter with Uranus in late January 1986 should resolve the question of the existence of new moons and reveal further structure within the rings.

\section{ACKNOWLEDGEMENTS}

It is a pleasure to thank Drs. W. F. Huebner and C. W. Patterson for their comments regarding this manuscript. 


\section{REFERENGES}

Alfvén, H. 1983, Astrophys. Space Sci. 97, 79.

Alfven, H, and Arrhenius, G. 1975, Structural And Evolutionary History of the Solar System, D, Reidel Publ. Co., Dordrecht, Holland.

Alfvên, H. and Arrhenius, G. 1976, Evolution of the Solar System, NASA SP-345, U. S, Government Printing Office, Washington, D.C.

Borderies, N. 1984, Celestial Mechanics 34, 297.

Curtis, S. A. and Ness, N. F. 1985, Geophysical Research Letters 12, 855.

E1liot, J. L., Dunham, E., and Mink, D. 1977, Nature 267, 328.

Elliot, J. L., Dunham, E., Wasserman, L. H., and Millis, R. L. 1978, Astronomical Journal $\underline{8} 3,980$.

Goldreich, P. and Tremaine, S. 1978, Icarus 34, 240.

Goldreich, P. and Tremaine, S. 1979, Nature 277, 97.

Holberg, J. B., Forrester, W. T., and Lissauer, J. J. 1982, Nature 297, 115.

Lissauer, J. J. and Cuzzi, J. N. 1982, Astronomical Journal 87, 1051.

Patterson, C. W. 1986, "Resonance Capture and Saturn's Rings," submitted to Icarus.

Prentice, A. J. R. 1986, "Formation of the Uranian System: pre-Voyager 2 Predictions," late paper presented at the 167 th Meeting of the American Astronomical Society.

Smith, B. A. 1985, Astronomicheskii Vestnik 19, 42.

Synnott, S. P., Terrile, R. J., Jacobson, R. A., and Smith, B. A. 1983, Icarus $\underline{53}, 156$. 


\section{TABLE 1}

ORBITAL RADII FOR HYPOTHETICAL SATELLITES

\begin{tabular}{|c|c|c|c|c|c|}
\hline Ring & $r_{\text {ring }}(\mathrm{km})$ & $r_{\min }(\mathrm{km})$ & $r_{\text {sat }}(\mathrm{km})$ & $r_{\text {sat }}\left(R_{u}\right)$ & Name \\
\hline \multirow[t]{2}{*}{6} & 41980 & & & & \\
\hline & & 42170 & 65891 & 2.52 & U1 \\
\hline \multirow[t]{2}{*}{5} & 42360 & & & & \\
\hline & & 42512 & 66424 & 2.54 & $\mathrm{U} 2$ \\
\hline 4 & 42663 & 43754 & 68365 & 2.61 & U3 \\
\hline \multirow[t]{2}{*}{$\alpha$} & 44844 & & & & \\
\hline & & 45322 & 70815 & 2.70 & U4 \\
\hline \multirow[t]{2}{*}{$\beta$} & 45799 & & & & \\
\hline & & 46561 & 72752 & 2.78 & U5 \\
\hline \multirow[t]{2}{*}{$\eta$} & 47323 & & & & \\
\hline & & 47534 & 74273 & 2.84 & U6 \\
\hline \multirow[t]{2}{*}{$\gamma$} & 47746 & & & & \\
\hline & & 48084 & 75132 & 2.87 & U7 \\
\hline$\delta$ & & 49730 & 77702 & 2.97 & U8 \\
\hline \multirow[t]{2}{*}{$\varepsilon_{i}$} & 51036 & & & & \\
\hline & 51752 & 51394 & 80303 & 3.06 & U9 \\
\hline
\end{tabular}

Notes:

Co1. 1: Ring destination assigned by E11iot et al. 1978. Upper and lower limits used for $\varepsilon$ ring.

Col. 2: Average ring radii given by Elliot et al. 1978 .

Col. 3: Adopted midpoint radii of ring minima.

Co1. 4: Predicted orbital radii for new satellites.

Col. 5: Same as Col. 4 but scaled to radius of Uranus $\left(R_{u}=26,200 \mathrm{~km}\right)$.

Col. 6: Tentative designation of new satellite. 


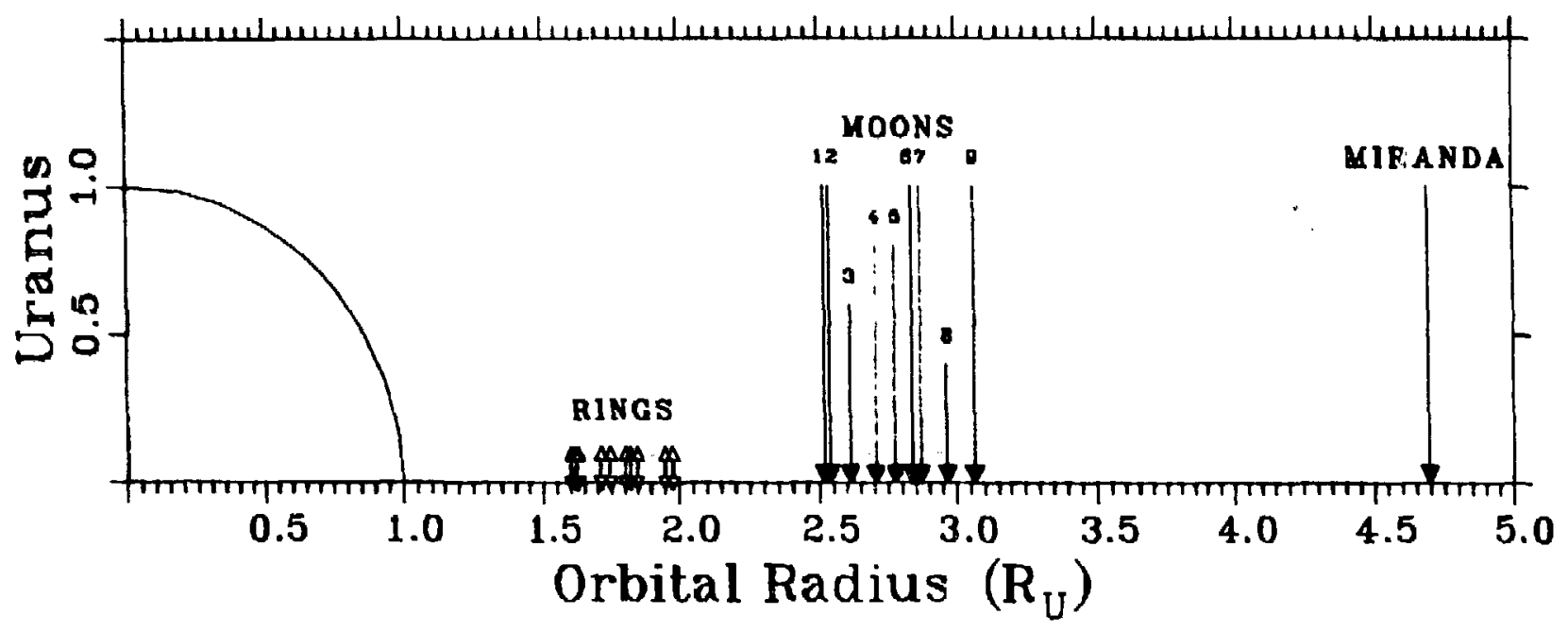

Figure 1. The location of the hypothetical moons in the Uranian system. These moons have been provisionally labeled U1 through U9. The relative error in the orbital radii is $\pm 0.04 R_{u}$. The radius of Uranus was adopted to be $26,200 \mathrm{~km}$. 